\title{
Activating PIK3CA mutation promotes osteogenesis of bone marrow mesenchymal stem cells in macrodactyly
}

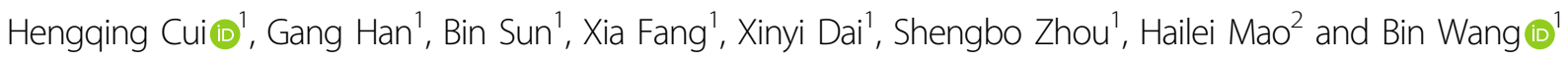

\begin{abstract}
Macrodactyly is a disabling congenital disease characterized by overgrowth of soft tissues and bones, which leads to finger enlargement and joint deformity. The mechanism of bone overgrowth in macrodactyly was rarely understood. In our study bone manifestations of three macrodactyly patients were analyzed by micro-CT. PIK3CA mutation was detected by next-generation sequencing (NGS) of a tumor gene-panel. The PI3K/AKT/mTOR pathway activation and target genes were analyzed. The osteogenic potential of macrodactyly-derived bone marrow mesenchymal stem cells (MAC-BMSCs) was compared with polydactyly-derived bone marrow mesenchymal stem cells (PD-BMSCs). PIK3CA inhibitors were tested for proliferation and osteogenesis potential of MAC-BMSCs. Activating PIK3CA mutations and activation of PI3K/AKT/mTOR pathway were detected in all MAC-BMSCs. MAC-BMSCs had enhanced osteogenesis potential compared with PD-BMSCs. PIK3CA knockdown by shRNA or BYL719 treatment significantly reduced osteogenic differentiation capacity of MAC-BMSCs. RNA-Seq and GRT-PCR revealed the upregulation of distal-less homeobox 5 (DLX5) in MAC-BMSCs compared with PD-BMSCs. The osteogenic potential of MAC-BMSCs was inhibited by DLX5 knockdown, indicating that DLX5 is a downstream target of PIK3CA activation-mediated osteogenesis. This study revealed that osteogenic differentiation in MAC-BMSCs is enhanced by PIK3CA activation mutation through PI3K AKT/mTOR signaling pathway and can be reversed by PIK3CA knockdown or drug inhibition.
\end{abstract}

\section{Introduction}

Macrodactyly is a rare congenital condition characterized by overgrowth of fingers or toes. It is a disabling disease, in which limb function and appearance are severely impaired. Overgrowth of bone, in addition to soft tissues, is often observed in macrodactyly and leads to enlarged finger size, abnormal bone formation and/or oblique fingers due to joint deformity. Oftentimes there is nerve involvement in the affected digits, among which median nerve is the most frequently involved ${ }^{1}$. Aberrant

\footnotetext{
Correspondence: Hailei Mao (mao.hailei@zs-hospital.sh.cn) or

Bin Wang (wangbin1766@163.com)

'Department of Plastic and Reconstructive Surgery, Shanghai Ninth People's Hospital, Shanghai JiaoTong University School of Medicine, Shanghai, China ${ }^{2}$ Department of Anesthesiology and Critical Care Medicine, Zhongshan

Hospital, Fudan University, Shanghai 200032, China

These authors contributed equally: Hengqing Cui, Gang Han

Edited by Y. Wang
}

distribution of neurofilament has been reported in the affected nerve tissues ${ }^{2}$. Osseous enlargement, osteochondromatous proliferations, hypertrophic changes, and ankylosis of innervated joints can be observed in some areas of innervated bone tissue ${ }^{3}$. Mutation of PIK3CA can also be detected in the diseased nerve tissue ${ }^{4}$. To date, debulking surgery is the only effective intervention. However, although dubulking surgery of soft tissue enlargement could achieve satisfactory outcomes ${ }^{5}$, overgrowth of bone created greater challenge and often resulted in amputation in severe cases ${ }^{6}$. The pathogenesis of bone hyperplasia needs to be further studied. Bone marrow stem cells (BMSCs) are a type of precursor cells that have the potential to differentiate into osteoprogenitor cells and therefore are of great importance for bone homeostasis ${ }^{7}$. The destruction of osteogenic differentiation of BMSCs may lead to the imbalance of bone 
homeostasis ${ }^{8}$. Yang et al. found that macrodactyly-derived adipose mesenchymal stem cells have significantly increased capability of osteogenic differentiation ${ }^{9}$. However, the role of BMSCs in pathological conditions remains largely unknown.

In this study, we first detected PIK3CA somatic mutations in MAC-BMSCs. We investigated the role of PI3K/ AKT/mTOR signaling pathway in osteogenic differentiation of MAC-BMSCs and explored the use of shRNA and p110 $\alpha$-specific inhibitor to block the osteogenic differentiation. Our study suggested DLX5 as a potential biomarker for development of potential therapeutic treatment for macrodactyly.

\section{Methods}

\section{Sample collection}

Surgically amputated digits were collected from three patients with isolated macrodactyly and three patients with polydactyly in Department of Plastic and Reconstructive Surgery, Shanghai 9th People's Hospital, followed by isolation of bone marrow mesenchymal stem cells (BMSCs). The detailed clinical information was listed in Table S1. This study was approved by the ethics committee of Shanghai 9th People's Hospital (reference: 201580). Written informed consent of sample collection and photograph was obtained.

\section{DNA isolation and sequencing}

Genomic DNA was extracted from bone marrow of amputated digits using QIAamp DNA Mini Kit (Qiagen, Valencia, CA, USA). DNA concentrations were measured with Qubit dsDNA HS assay kit (Life Technologies) on NanoDrop spectrophotometer (Thermo Fisher Scientific). Genomic DNA integrity was determined by agarose gel electrophoresis.

For targeted next-generation sequencing, genomic DNA was sheared to an average size of $200 \mathrm{bp}$ using a Covaris S220 series sonicator. Fragmentation was followed by library construction with KAPA Hyper Prep kit (KAPA Biosystem, Roche) containing mixes for end repair, Atailing and adaptor ligation. Prepared library was hybridized for 16-24h using custom capture DNA probes (Integrated DNA Technology Inc.). Target exons were captured by a panel designed by Shanghai Sinomics Corporation covering the coding exons of 593 genes which relate to clinical target therapy and pathogenetic mechanism of cancer (Table S2). The hybridized product was captured by streptavidin beads (Invitrogen) and amplified for 12 PCR cycles using KAPA HiFi HotStart ReadyMix. The amplified products were quantified by Qubit ${ }^{\circledR}$ 2.0 Fluorometer (Life Technologies, USA) and validated by Agilent 2100 bioanalyzer (Agilent Technologies, USA) to confirm the insert size and the mole concentration.
DNA sequencing was performed on HiSeq X-Ten sequencing system (Illumina) with $2 \times 150 \mathrm{bp}$ pair end sequencing.

Mutations detected in next generation were confirmed by Sanger sequencing. Primer sequences and additional details for polymerase chain reaction and Sanger sequencing are available upon request.

\section{Cell culture}

BMSCs were isolated from amputated digits as described previously ${ }^{10}$. Briefly, bone marrow cells were washed out of the phalanx bones and centrifuged at $1000 \times g$ for 5 min. Cells were cultured in HBMSCs medium (Cyagen, CA, USA) containing $10 \%$ fetal bovine serum, $1 \%$ penicillin and streptomycin, and maintained in a humidified atmosphere containing $5 \% \mathrm{CO}_{2}$ at $37^{\circ} \mathrm{C}$. BMSCs were digested with $0.25 \%$ trypsin and passaged routinely when 80-90\% confluence was reached. BMSCs from passages 3-6 were used in the following experiments.

\section{Cell proliferation assay}

Both PD-BMSCs and MAC-BMSCs were seeded in 96well plates at a density of 2000 cells per well. Cell proliferation assay was performed on days $1,3,5$, and 7 using cell counting kit 8 (CCK-8, Dojindo, Japan), following the manufacture's instruction. Briefly, $10 \mu \mathrm{L}$ CCK- 8 was added to each well and incubated for $2 \mathrm{~h}$ at $37^{\circ} \mathrm{C}$. The absorbance was measured at $562 \mathrm{~nm}$ using a microplate reader (Bio-Rad, Hercules, CA, USA). All experiments were repeated at least three times.

\section{Osteogenic differentiation}

Osteogenic differentiation was conducted as previously described $^{11}$. Briefly, MAC-BMSCs and PD-BMSCs were placed in a 12-well plate at a density of $5 \times 10^{4}$ cells per well. Osteogenic differentiation was induced in basic growth medium supplemented with $100 \mathrm{nM}$ dexamethasone (Cyagen, CA, USA) and $50 \mu \mathrm{M}$ ascorbic acid (Cyagen, CA, USA). Osteogenic media was changed every 3 days. Quantitative real-time PCR examination of osteogenic marker genes were conducted 7 days after induction.

\section{Alkaline phosphatase (ALP) analysis and Alizarin red staining}

ALP staining was performed 7 days after osteogenic induction according to the manufacturer's instruction (Beyotime, Shanghai, China). Mineralized nodule formation was detected by $2 \%$ Alizarin red (Cyagen, CA, USA) 14 days after osteoblast differentiation. ALP activity of BMSCs was measured by a colorimetric enzymatic assay of alkaline phosphatase following the manufacturer's protocol (Jiancheng, Nanjing, China). ALP activity was normalized to the total protein content determined by a 
bicinchoninic acid (BCA) kit (Thermo Fisher Scientific, USA). To quantify calcium accumulation, alizarin red was eluted by $10 \%$ cetylpyridinium chloride (CPC; Sigma) and absorbance at $570 \mathrm{~nm}$ was measured.

\section{Adipogenic differentiation}

Adipogenic differentiation was conducted following the manufacturer's instruction (Cyagen, CA, USA). Briefly, the MAC-BMSCs and PD-BMSCs were seeded in growth medium at a density of $5 \times 10^{4}$ per well in a 12-well plate. The growth medium was changed every 3 days until the cells become fully confluent. The cells were incubated in adipogenic differentiation medium A (induction medium) for 3 days and the medium was replaced by adipogenic differentiation medium B (maintenance medium). After $24 \mathrm{~h}$, the medium was changed back to adipogenic differentiation medium A. After the cycle of induction and maintenance was repeated for four times, cells were maintained in adipogenic differentiation medium $B$ for additional 6 days until the lipid droplets are big and round enough. During this period, medium was changed every 3 days.

\section{Oil red $O$ staining analysis}

After the cells have differentiated, the adipogenic differentiation medium was removed. The cells were rinsed with phosphate-buffered saline (PBS) and fixed with $4 \%$ formaldehyde solution for $30 \mathrm{~min}$. The cells were rinsed twice with $1 \times \mathrm{PBS}$ and stained with $0.5 \mathrm{~mL}$ of oil red $\mathrm{O}$ working solution (3:2 dilution with distilled water and filter with filter paper) for $30 \mathrm{~min}$. The wells were rinsed with $1 \times$ PBS for three times. Cells were visualized and analyzed under a microscope.

\section{RNA extraction and quantitative reverse transcription polymerase chain reaction (qRT-PCR)}

Total RNA was extracted using the TRIzol reagent (Invitrogen, Carlsbad, CA, USA). The reverse transcription reaction was prepared on ice following the instructions of PrimeScript RT reagent Kit (TaKaRa, Shiga, Japan), and cDNA was obtained after the reaction completion. Quantitative RT-PCR was then performed by using Thunderbird SYBR qPCR Mix (Toyobo, Japan), with $10 \mu \mathrm{L}$ of the total reaction system. The PCR amplification conditions were: predenaturation at $95^{\circ} \mathrm{C}$ for $2 \mathrm{~min}$, followed by 40 cycles of $95^{\circ} \mathrm{C}$ for $20 \mathrm{~s}, 60^{\circ} \mathrm{C}$ for $20 \mathrm{~s}$, and $72{ }^{\circ} \mathrm{C}$ for $20 \mathrm{~s}$. The primer sequences were listed in Table S3. The relative quantification of gene expression was analyzed with the values of $2^{-\Delta \Delta C T}$, normalized with GAPDH expression level. Triplicate tests were conducted in each experiment.

\section{Western blot analysis}

Cells were lysed by RIPA Lysis Buffer (Beyotime, China) supplemented with phosphatase inhibitor for total protein extraction. The concentration of each protein sample was quantified by a BCA (bicinchoninic acid) kit (Thermo Fisher Scientific, USA). Equal amounts of protein were separated by gel electrophoresis and then transferred to PVDF (polyvinylidene difluoride) membranes (Millipore, Billerica, MA, USA). After being blocked with $5 \%$ bovine serum albumin (BSA) in TBST for $30 \mathrm{~min}$ at room temperature, the PVDF membrance was incubated with primary antibodies (catalog numbers are listed in Table S3) at $4{ }^{\circ} \mathrm{C}$ overnight. After washing three times in TBST for $5 \mathrm{~min} /$ wash, the membranes were incubated with HRPconjugated secondary antibody (Beyotime, A0208, 1:8000) for $45 \mathrm{~min}$ at room temperature. Protein bands were exposed via enhanced chemiluminescence (ECL, Thermo Fisher Scientific, Inc.) method.

\section{Knockdown of PIK3CA and DLX5 expression by short hairpin RNA}

MAC-BMSCs were seeded at $3 \times 10^{5}$ cells per well into a 6-well plate and allowed to settle overnight. BMSCs were infected with lentivirus $(\mathrm{MOI}=40)$ for $3 \mathrm{~h}$ in the presence of polybrene $(8 \mu \mathrm{g} / \mathrm{ml})$ and then maintained in regular medium. Forty-eight hours later, $4 \mu \mathrm{g} / \mathrm{ml}$ of puromycin was added and cells were cultured until all cells in the control group were dead. PIK3CA expression and $\mathrm{PI} 3 \mathrm{~K} / \mathrm{AKT} / \mathrm{mTOR}$ suppression were analyzed 3 days after puromycin selection.

\section{BYL719 administration}

MAC-BMSCs were treated with BYL719 (Selleck, Houston, TX, USA) at the dose of $1.25,2.5$, or $5 \mu \mathrm{M} 24 \mathrm{~h}$ after cell attachment. The protein expression of PI3K/ AKT/mTOR pathway was detected by western blotting $2 \mathrm{~h}$ after treatment.

\section{RNA sequencing and data analysis}

Total RNAs was extracted from passage 3 of cultured MAC-BMSCs $(n=2)$ and PD-BMSCs $(n=2)$ by using RNeasy mini kit (Qiagen, Germany). Complementary DNA library preparation and sequencing were performed according to Illumina standard protocol. Raw data of gene expression profiles of human bone marrow stem cells derived from macrodactyly and polydactyly patients, were submitted to the GEO database (https://www.ncbi.nlm. nih.gov/geo/) and the Geo accession number is GSE 147823. Gene Ontology (GO) analysis was performed with DAVID online tool. Top GO categories were selected according to the $p$ values of expression changes. Eight differentially expressed genes were randomly selected and validated by qRT-PCR. The primers are listed in Table S4.

\section{Statistics analysis}

Data were showed as mean \pm standard deviations from at least three independent experiments. Differences 


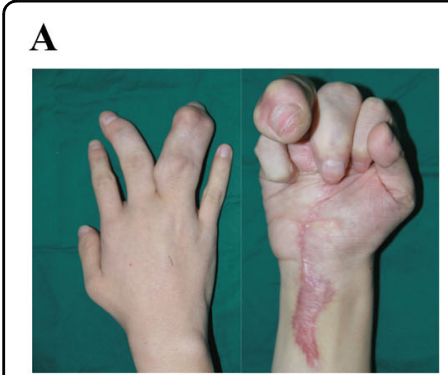

B

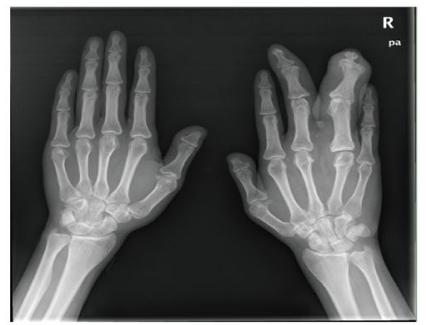

C

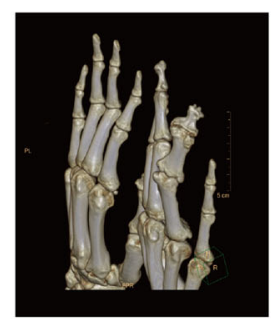

D

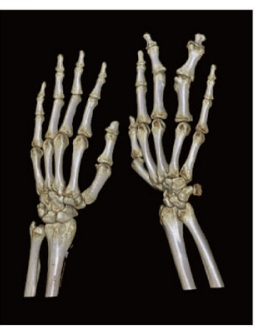

$\mathbf{E}$

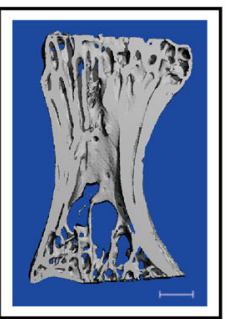

F MAC

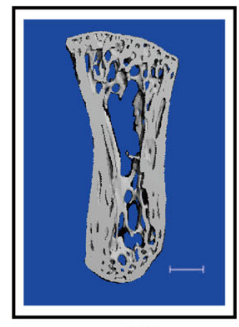

PD
G

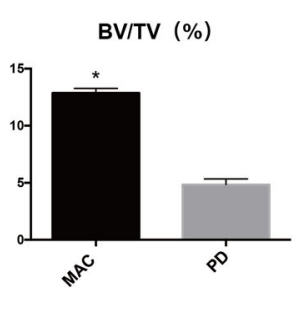

I

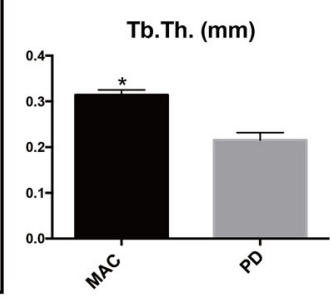

H
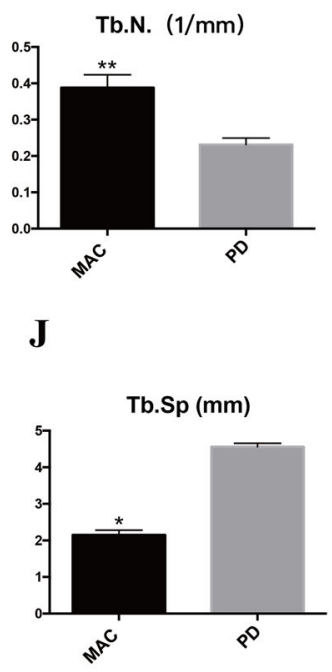

Fig. 1 Clinical manifestation in patients with macrodactyly. a A macrodactyly patient with enlarged middle and ring fingers. b Radiograph demonstrating the hyperplastic bone formation compared with the normal hand. c, d Computed tomography (CT) with 3D reconstruction. e, $\mathbf{f}$ Micro-CT images of phalanxs from macrodactyly (MAC) and polydactyly (PD) patients (scale bar $=1.0 \mathrm{~mm}$ ). $\mathbf{g}-\mathbf{j}$ Quantitative parameters of Micro-CT. BV/TV: bone volume/total volume, Tb.N.: trabecular number, Tb.Th.: trabecular thickness, Tb.Sp: trabecular separation (g, h, i, $\mathbf{j}$ two-tailed unpaired $t$-test, $\left.{ }^{*} p<0.05,{ }^{* *} p<0.01\right)$.

between groups were analyzed using one-way analysis of variance (ANOVA) followed by Tukey test. Data were considered statistically significant when $p$ value is $<0.05$. All statistical analysis was performed by Prism software (GraphPad Software, La Jolla, CA, USA).

\section{Results}

Bone overgrowth in macrodactyly patients

Radiographic examination of macrodactyly patient \#1 showed enlarged bone volume as well as increased soft tissue volume in the right ring finger (Fig. 1a). The $\mathrm{X}$-ray plain film revealed that the bone volume of the middle finger and ring finger of the right hand increased, and the bone mineral density was higher than that of the healthy fingers (Fig. 1b). CT three-dimensional reconstruction showed that there were joint deformities in the proximal joint of the middle and ring fingers as well as oblique finger deformities on the right hand (Fig. 1c, d). Micro-computed tomography (micro-CT) examination indicated an approximately twofold increase of bone volume fraction (BV/TV) in the macrodactylous phalanx compared with the phalanx of polydactyly (Fig. 1g). In addition, macrodactylous bones showed increased trabecular number (Tb.N., Fig. 1h), trabecular thickness (Tb.Th., Fig. 1i) and reduced trabecular separation (Tb.sp., Fig. 1j). These data demonstrated bone hyperplasia in macrodactyly.

\section{PIK3CA-mutated MAC-BMSCs displayed increased proliferation and osteogenesis potential}

To investigate whether BMSCs play a role in bone overgrowth in macrodactyly, we isolated BMSCs from amputated macrodactylous digits and compared with BMSCs from polydactyly patients. MAC-BMSCs (Fig. 2a) and PD-BMSCs (Fig. 2b) showed similar morphology in culture and expressed identical BMSC markers (Fig. 2c). We identified a somatic mutation of PIK3CA (c.3140A $>$ G, p.H1047R) in MAC-BMSCs, but not in PD-BMSCs (Fig. 2d). Patients' detailed information is listed in supplementary Table S1. Phosphorylation of AKT and mTOR was significantly increased in passage 3 MAC-BMSCs (Fig. 2e), indicating enhanced activity of the PI3K/AKT/ mTOR signaling pathway.

Furthermore, MAC-BMSCs demonstrated higher osteoblast differentiation potential than PD-BMSCs when subjected to osteogenic induction, as indicated by increased Alkaline phosphatase (ALP) staining and Alizarin red staining (Fig. 3a). The quantitative analysis of the staining results revealed a significant difference between the two groups (Fig. 3b, c). Consistently, expression of osteoblast marker genes collagen 1 1 (Col1a1), ALP, and Runx2 was increased in differentiated MAC-BMSCs (Fig. 3d). The cell growth curve showed that the proliferation rate of MAC-BMSCs was significantly higher than that of PD-BMSCs (Fig. 3e). 


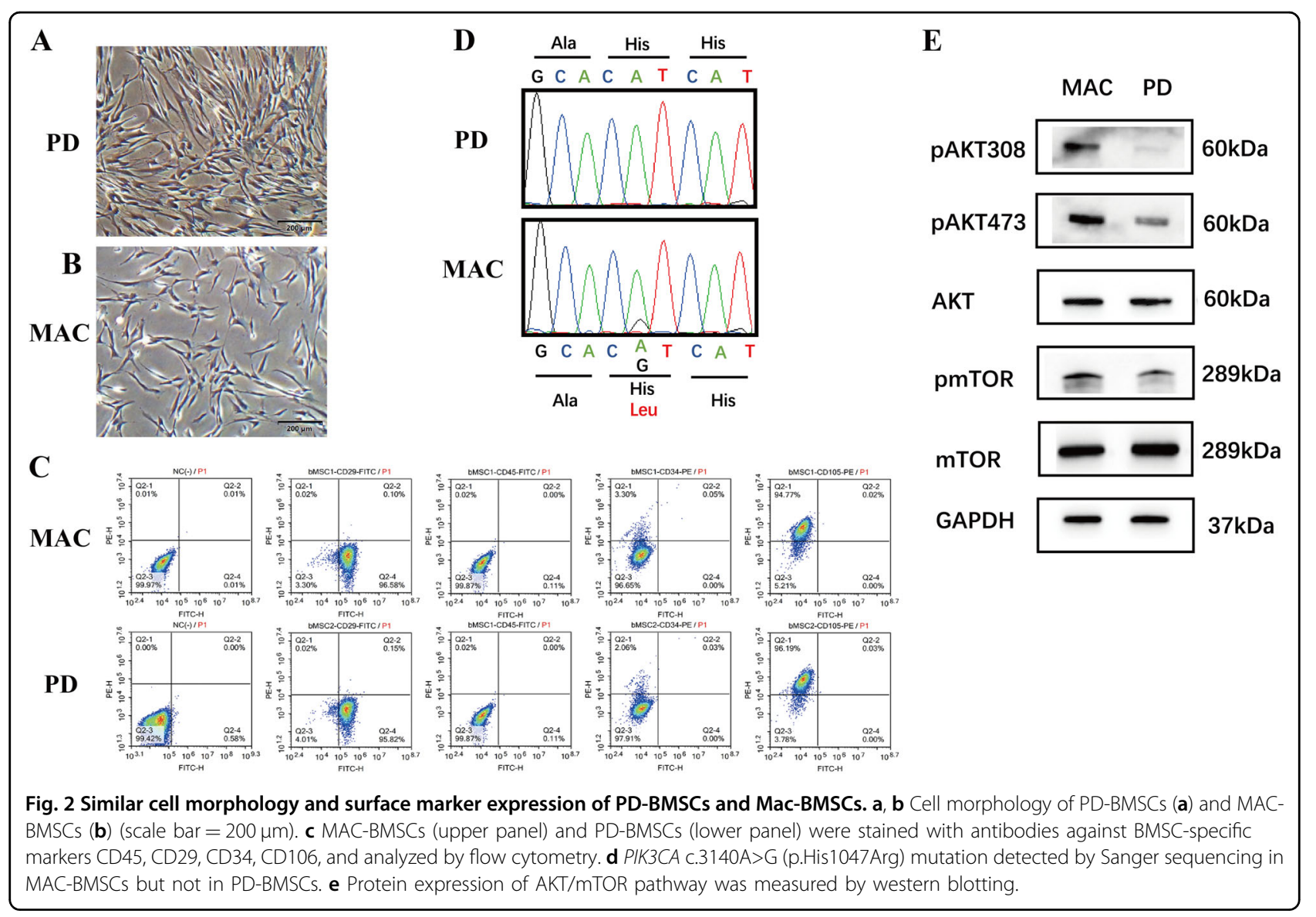

Compared with wild-type cells, the adipogenic differentiation ability of PIK3CA mutant cells was significantly enhanced (Fig. 3f).

\section{Knockdown of PIK3CA reduced osteogenesis potential of MAC-BMSCs}

$\mathrm{PI} 3 \mathrm{~K} / \mathrm{AKT} / \mathrm{mTOR}$ signaling pathway has been positively correlated with osteogenesis ${ }^{12}$. To investigate whether enhanced osteogenesis of MAC-BMSCs resulted from elevated PI3K signaling upon PIK3CA hyperactivation, we knocked down PIK3CA by lentivirus-mediated shRNA transfection in MAC-BMSCs. Decreased PIK3CA expression was confirmed by qRT-PCR (Fig. 4a) and western blot analysis (Fig. 4f). The intensity of ALP staining and Alizarin red staining in the PIK3CA knockdown group was much lower than the scramble RNA control group (Fig. $4 \mathrm{~b})$, and the quantitative analysis confirmed the statistical differences between the two groups (Fig. 4c, d). The osteogenic marker genes were also significantly downregulated in the knockdown group (Fig. 4e). These results showed that knocking down PIK3CA decreased the osteogenic potential of MAC-BMSCs, indicating an essential role of PIK3CA activity in regulating osteogenic differentiation of BMSCs in macrodactyly.

\section{Activated PI3K pathway enhanced osteogenic differentiation of PD-BMSCs}

To further investigate if PIK3CA activation leads to elevated osteogenic potential of BMSCs, we activated PI3K signaling pathway in PD-BMSCs with IGF-1. Indeed, IGF-1 treatment promoted AKT phosphorylation (Fig. 5a) and osteogenesis of PD-BMSCs. Importantly, this effect can be greatly reversed by BYL719, an aspecific PI3K inhibitor. ALP and Alizarin red staining were significantly enhanced by IGF-1 in PD-BMSCs and the enhanced staining was significantly attenuated by BYL719 (Fig. 5b-d). Consistently, the expression of osteogenic marker genes ALP, Runx2, and Col1 1 1 was increased by IGF-1 and inhibited by BYL719 (Fig. 5e), suggesting that the ability of osteogenesis induction by PIK3CA activation could be reversed by BYL719.

\section{BYL719 inhibited osteogenic potential of MAC-BMSCs}

To look into the possibility of suppressing osteogenesis potential of MAC-BMSCs by pharmaceutical inhibition agent targeting at PIK3CA, we treated differentiating MAC-BMSCs with BYL719. BYL719 treatment inhibited phosphorylation of AKT and mTOR in a dose-dependent manner, suggesting effective inhibition of p110 $\alpha$ activity 

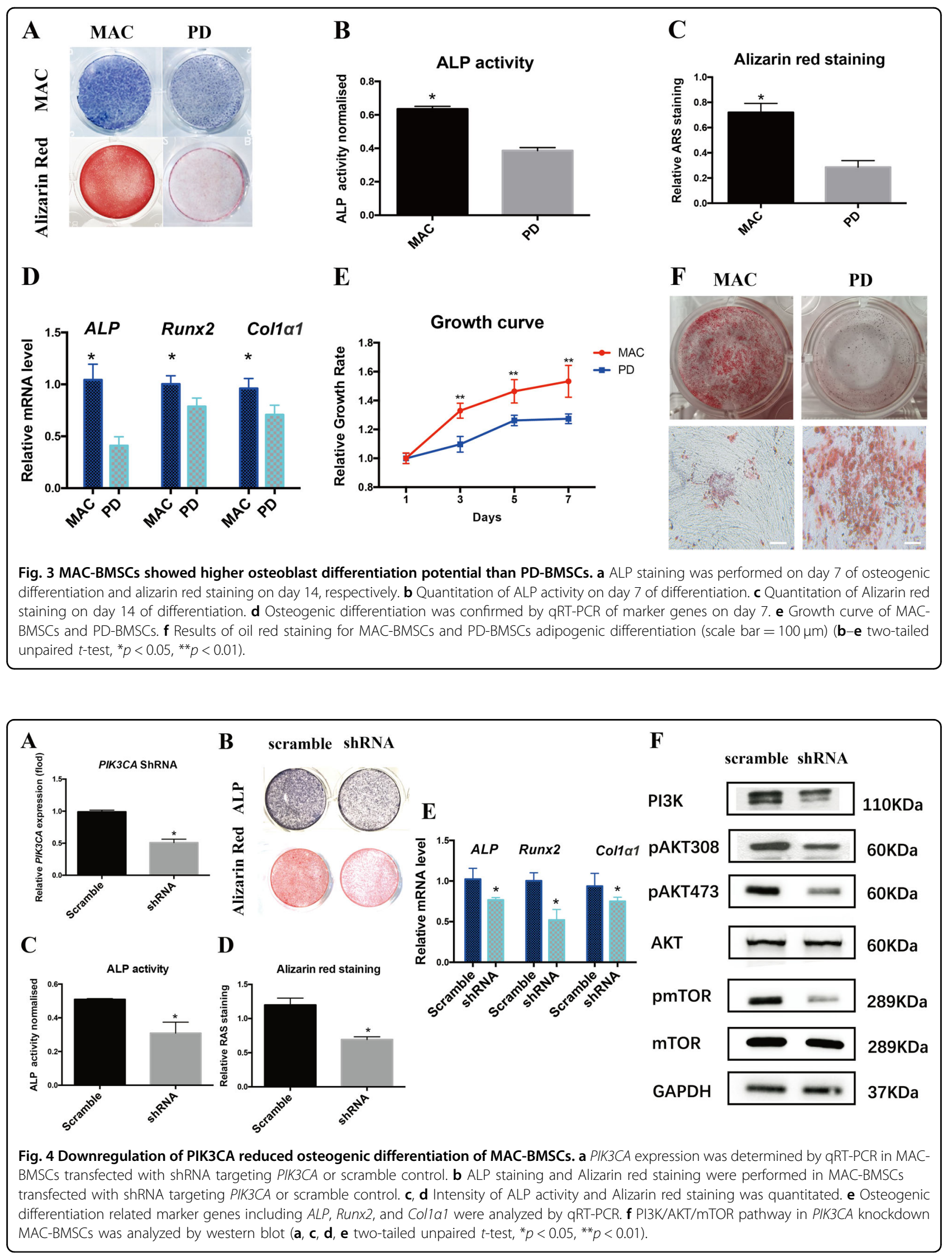


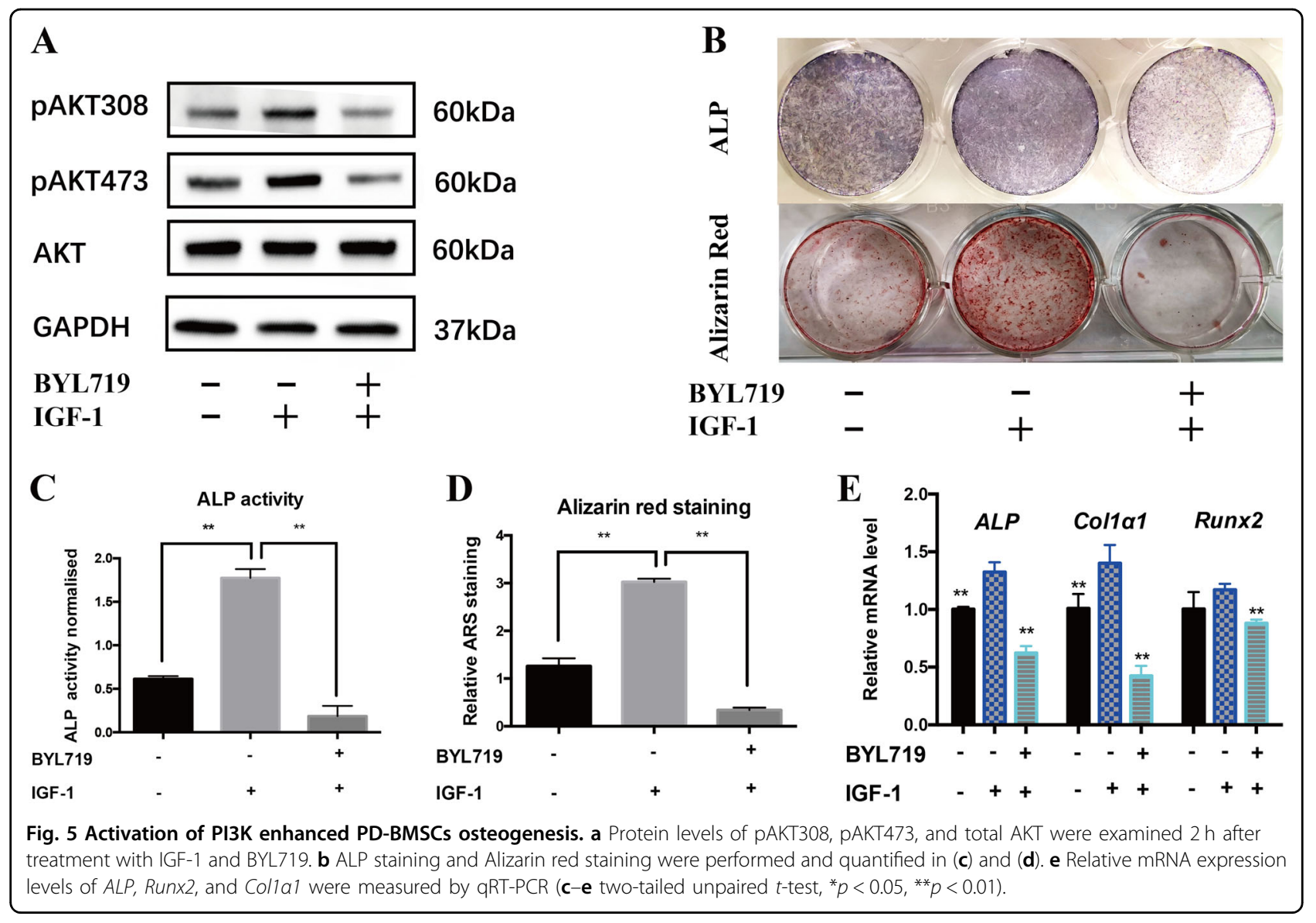

(Fig. 6a). Consistently, BYL719 treatment resulted in decreased osteogenic differentiation of MAC-BMSCs in a dose-dependent manner, as shown by quantitative analysis of alkaline phosphatase staining (ALP) (Fig. 6c), alizarin red staining (Fig. 6d), and mRNA expression of Col1a1, ALP, and Runx2 genes (Fig. 6e).

\section{PIK3CA regulated osteogenic differentiation via DLX5}

To understand the mechanism of PIK3CA-mediated regulation of osteogenesis, we performed RNA-Seq analysis of PD-BMSCs and MAC-BMSCs. Differential expression analysis revealed that 383 genes were upregulated and 264 genes were downregulated in MACBMSCs versus PD-BMSCs $(\operatorname{logFC}<1, p<0.01, p$-adj $<$ 0.01) (Fig. 7a). Eight differentially expressed genes were randomly selected for validation by $\mathrm{qRT}$-PCR as shown in Fig S1. Six of the 383 upregulated genes were associated with osteoblast differentiation. However, among the six genes, only $D L X 5$ was downregulated upon PIK3CA knockdown in MAC-BMSCs (Fig. 7b). The mRNA expression level of $D L X 5$ was also significantly higher in MAC-BMSCs $(n=6)$ than in PD-BMSCs $(n=6)$ (Fig. 7c). In the $D L X 5$ knockdown experiment, mRNA expression of $D L X 5$ was inhibited by the transfection of $D L X 5$ lentivirus shRNA (Fig. 7d), the expression level in Sh\#3 was reduced to $16 \%$ of the control group. The protein expression of $D L X 5$ was significantly decreased at the same time (Fig. 7e). In the DLX5 knockdown MACBMSCs, the osteogenic induction and differentiation ability of MAC-BMSCs was significantly decreased, as indicated by ALP staining and Alizarin red staining (Fig. 7f) and osteogenic marker gene expression analysis (Fig. $7 \mathrm{~g})$. The results of quantitative analysis also confirmed the above results (Fig. 7h, i). Collectively, these data suggest that $D L X 5$ regulation by $P I K 3 C A$ could play an important role in osteogenesis.

\section{Discussion}

Macrodactyly is one of the PIK3CA related overgrowth spectrum (PROS) caused by somatic mutation of PIK3CA $A^{13}$, which encodes the catalytic $\alpha$-subunit of PI3K (p110 $\alpha$ ). PI3K catalyzes the conversion of phosphatidylinositol-4,5-bisphosphate $\left(\mathrm{PIP}_{2}\right)$ to phosphatidylinositol $(3,4,5)$-triphosphate $\left(\mathrm{PIP}_{3}\right)^{14} \cdot \mathrm{PIP}_{3}$ recruits $\mathrm{AKT}$ and promotes PDK1 phosphorylation of AKT at Thr308 ${ }^{15}$. H1047R, E542K, and C420R mutations are located in the kinase domain, helical domain, and $\mathrm{C} 2$ domain, respectively. H1047R and E542K are 'hot-spot' mutations, and 

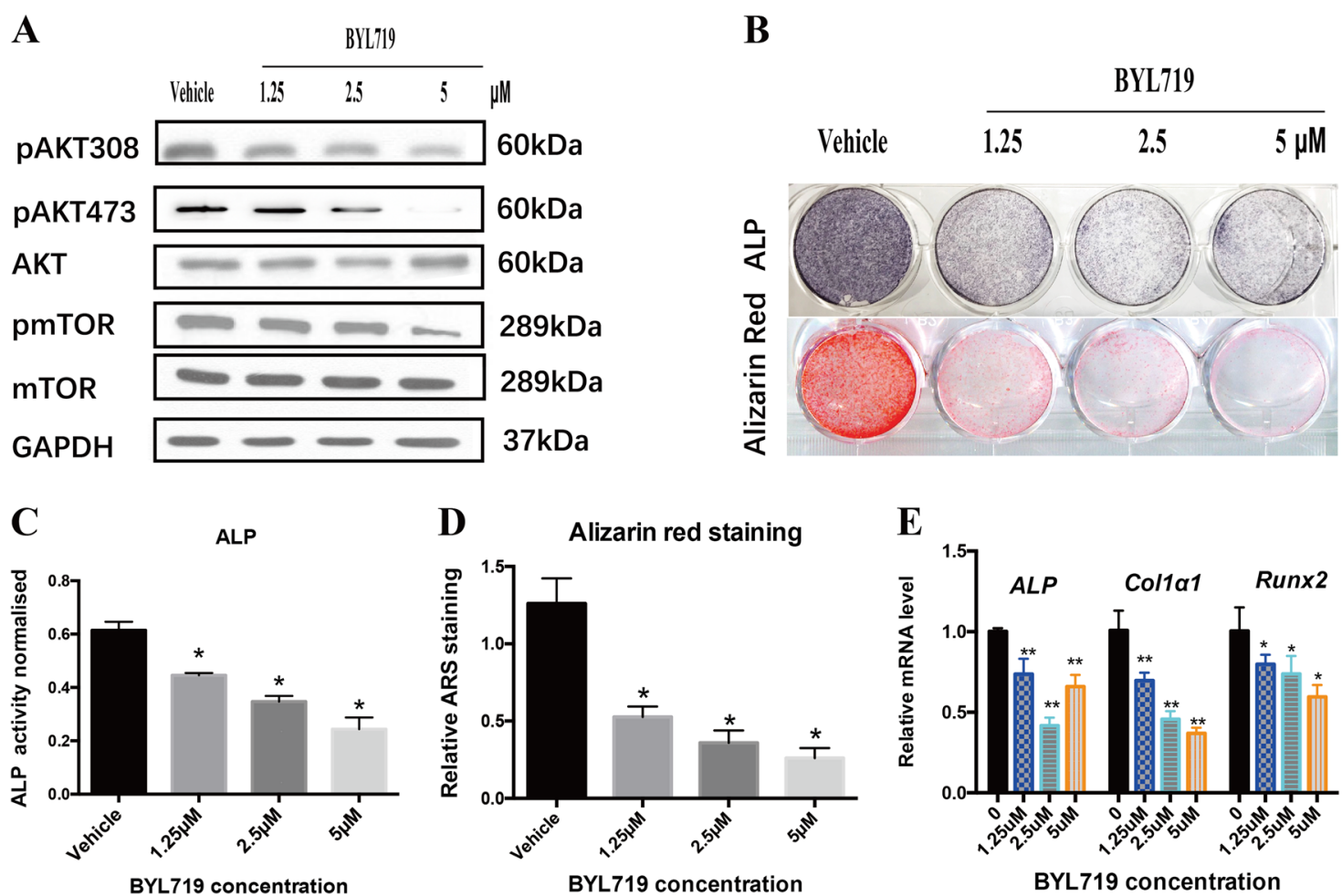

Fig. 6 Suppression of MAC-BMSCs osteogenesis by BYL719. a Protein levels of pAKT308, pAKT473, total AKT, pmTOR, and total mTOR of MACBMSCs were examined $2 \mathrm{~h}$ after treatment with BYL719. $\mathbf{b}$ ALP staining and Alizarin red staining were performed and quantified in (c) and (d). e Relative mRNA expression levels of $A L P, R u n \times 2$, and Col1a 1 were measured by qRT-PCR (c-e two-tailed unpaired $t$-test, ${ }^{*} p<0.05$, ${ }^{* *} p<0.01$ ).

C420R is frequently detected in macrodactyly. Many studies have shown that overexpression of PIK3CA with H1047R, E542K, and C420R point mutations in different cell types can induce a gain-of-function activation PI3KAKT-mTOR pathway and cause phenotypic changes such as enhanced proliferation and invasion ${ }^{16-19}$. We also demonstrated this in bone marrow mesenchymal stem cells derived from macrodactyly in our research. Although $\mathrm{PI} 3 \mathrm{~K} / \mathrm{AKT} / \mathrm{mTOR}$ cell-signaling pathway has been shown to play an important role in macrodactyly ${ }^{13,20,21}$, whether or not this pathway contributes to bone malformation in macrodactyly was unknown.

Overgrowth of bone in macrodactyly is a great challenge for hand surgeons.

In this study, we demonstrated that PIK3CA was mutated in BMSCs derived from overgrown bones in macrodactyly patients and the activating mutations resulted in hyperactivation of PI3K/AKT/mTOR signaling pathway and enhanced osteogenic differentiation. We further explored the downstream osteogenesis pathway and identified $D L X 5$ gene as a potential target gene. Bone overgrowth is a common feature in macrodactyly patients. Bone formation relies on BMSCs which give rise to new osteoblasts and their progenitors ${ }^{22}$. PI3K/AKT pathway has been reported to promote osteoblast differentiation and accumulation of bone $\mathrm{e}^{23-25}$. We showed that PIK3CA gene was mutated in MAC-BMSCs which resulted in hyperactivation of $\mathrm{PI} 3 \mathrm{~K} / \mathrm{AKT} / \mathrm{mTOR}$ pathway while no PIK3CA mutation was detected in PD-BMSCs. Importantly, MAC-BMSCs displayed higher osteogenic potential than PD-BMSCs. Consistent with the role of PI3K signaling in osteogenesis, MAC-BMSCs exhibited higher osteogenic potential than PD-BMSCs. We further demonstrated that knocking-down of PIK3CA in MACBMSCs led to inhibited osteogenesis while activation of PI3K signaling in PD-BMSCs resulted in enhanced osteogenesis. To our knowledge, this is the first study revealing the molecular mechanism of bone overgrowth in macrodactyly.

By comparing the transcriptome profiling of MACBMSCs versus PD-BMSCs, we identified DLX5 as a differentially expressed osteogenesis-related gene and the only gene that responded to PIK3CA knockdown among a series of osteogenic genes. DLX5 can bind to Runx2 enhancer and drive Runx2 expression, which is a key regulator of BMSC osteogenic differentiation ${ }^{26}$. Importantly, Dai et al. demonstrated that mTORC1-S6K1 regulates osteogenesis via $D L X 5$ regulation of Runx $2^{27}$. Therefore, DLX5 may serve as a downstream effector of PI3K-mediated hyper-osteogenesis of MAC-BMSCs. 


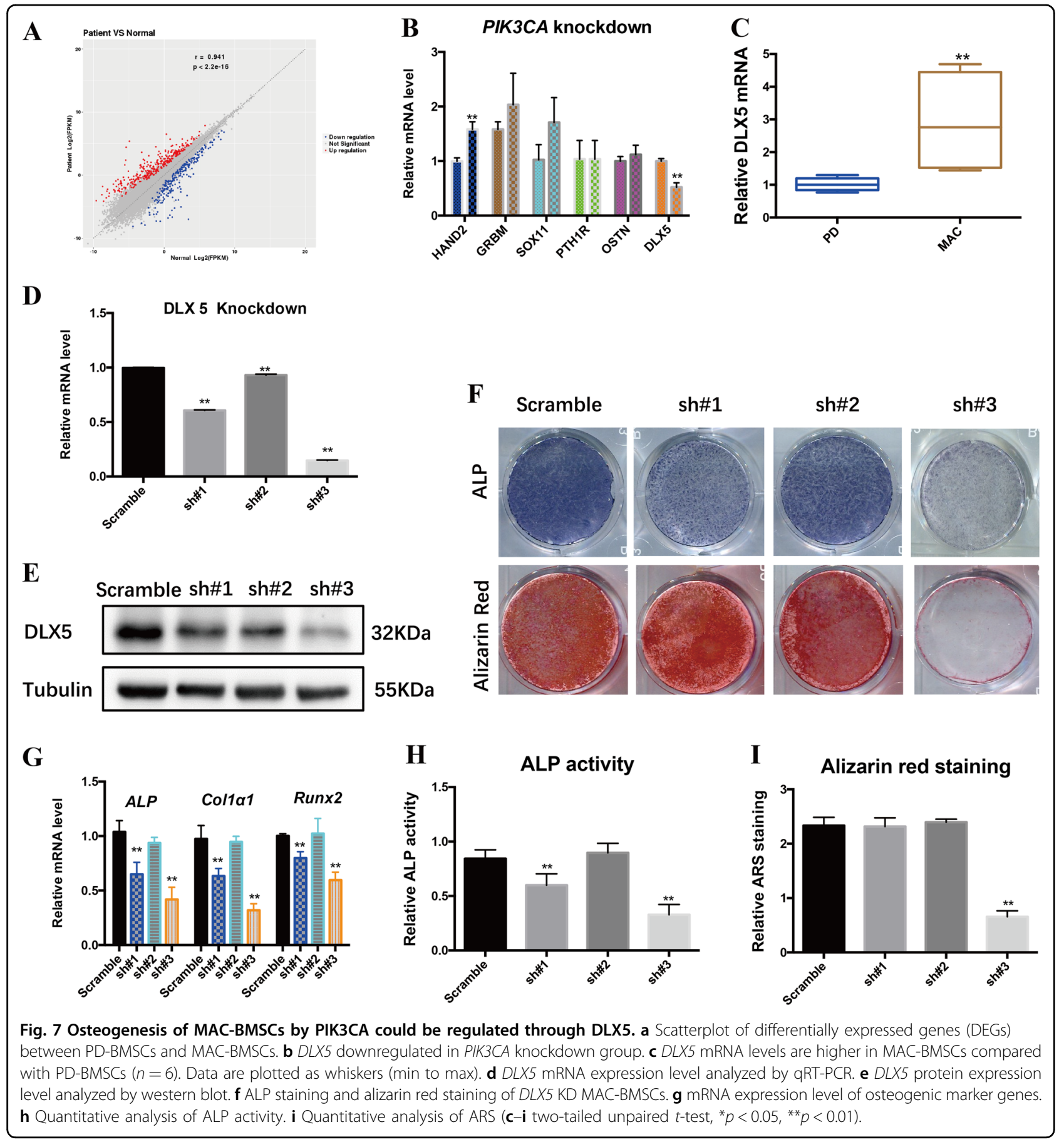

Understanding the involvement of PIK3CA hyperactivation in osteogenesis of MAC-BMSCs provides an opportunity to pharmaceutically inhibit the bone overgrowth in macrodactyly. BYL719 is a selective inhibitor for $\mathrm{p} 110 \alpha$ which has been approved to treat PIK3CAmutated breast cancer. Venot et al. administered BYL719 to 19 patients with PROS disorders, showing dramatic clinical improvement in suppressing tissue overgrowth and even in correcting the skeletal deformity ${ }^{21}$. Notably, recipients in the study displayed good toleration. Only three patients had herglycaemia and no other side effects were observed. This study suggests a therapeutic strategy to use BYL719 as a potential drug to prevent abnormal bone formation in macrodactyly. The impact of PIK3CA blockage on cell survival is inevitable ${ }^{28}$. The decrease in staining due to inhibition of cell proliferation was corrected by normalizing the quantity of alkaline phosphatase staining to total protein. The expression of osteogenic 
marker genes (alp, runx2, and col1 1 1) was evaluated to confirm the inhibitory effect of BYL719 on MAC-BMSCs osteogenesis. Our study demonstrated that the PIK3CAspecific small molecule inhibitor can effectively reduce osteogenesis of MAC-BMSCs, showing a promising therapeutic potential.

In summary, we reported for the first time the possible mechanism of hyperplastic bone formation in macrodactyly. We identified PIK3CA mutations in BMSCs from macrodactylous bones and demonstrated the role of PI3K/AKT activation in the osteogenesis of MACBMSCs. Our findings provide the theoretical basis for targeted therapy of macrodactyly by inhibiting key regulators such as PI3K and DLX5.

\section{Acknowledgements}

We would like to thank the families for their participation in this study. This study was supported by The National Key Research and Development Program of China (2018YFC1105800), National Natural Science Foundation of China (81772115, 81571930, and 51973041), and Shanghai Natural Science Foundation (19ZR1408800).

\section{Conflict of interest}

The authors declare that they have no conflict of interest.

\section{Publisher's note}

Springer Nature remains neutral with regard to jurisdictional claims in published maps and institutional affiliations.

Supplementary Information accompanies this paper at (https://doi.org/ 10.1038/s41419-020-2723-6).

Received: 8 December 2019 Revised: 18 June 2020 Accepted: 22 June 2020 Published online: 06 July 2020

\section{References}

1. Silverman, T. A. \& Enzinger, F. M. Fibrolipomatous hamartoma of nerve. A clinicopathologic analysis of 26 cases. Am. J. Surg. Pathol. 9, 7-14 (1985).

2. Yang, X. et al. Morphological and neurophysiological impairment of the nerve in type II macrodactyly. PLOS ONE 13, e0200183 (2018).

3. Blackburn, P. R. et al. PIK3CA mutations in lipomatosis of nerve with or without nerve territory overgrowth. Mod. Pathol. 33, 420-430 (2020).

4. Maclellan, R. A. et al. PIK3CA activating mutations in facial infiltrating lipomatosis. Plast. Reconstr. Surg. 133, 12e-19e (2014).

5. Rechnagel, K. Megalodactylism. Report of 7 cases. Acta Orthopaedica Scandinavica 38, 57-66 (1967).

6. Cerrato, F. et al. Presentation and treatment of macrodactyly in children. $J$. Hand Surg. 38, 2112-2123 (2013).
7. Crane, J. L. \& Cao, X. Bone marrow mesenchymal stem cells and TGF-beta signaling in bone remodeling. J. Clin. Invest. 124, 466-472 (2014).

8. Zaidi, M. Skeletal remodeling in health and disease. Nat. Med. 13, 791-801 (2007).

9. Yang, X. et al. Altered adipose-derived stem cell characteristics in macrodactyly. Sci. Rep. 7, 11090 (2017)

10. Benedict, M. R. et al. Dissociation of bone mineral density from age-related decreases in insulin-like growth factor-l and its binding proteins in the male rat. J. Gerontol. 49, B224-B230 (1994).

11. Greenblatt, M. B. et al. CHMP5 controls bone turnover rates by dampening NF-kappaB activity in osteoclasts. J. Exp. Med. 212 1283-1301 (2015).

12. Zhang, Z. et al. TGFbeta1 promotes the osteoinduction of human osteoblasts via the PI3K/AKT/mTOR/S6K1 signalling pathway. Mol. Med. Rep. 19, 3505-3518 (2019).

13. Rios, J. J. et al. Somatic gain-of-function mutations in PIK3CA in patients with macrodactyly. Hum. Mol. Genet. 22, 444-451 (2013).

14. Hiles, I. D. et al. Phosphatidylinositol 3-kinase: structure and expression of the 110 kd catalytic subunit. Cell 70, 419-429 (1992).

15. Stephens, L. et al. Protein kinase B kinases that mediate phosphatidylinositol 3,4,5-trisphosphate-dependent activation of protein kinase B. Science 279, 710-714 (1998).

16. Ikenoue, T. et al. Functional analysis of PIK3CA gene mutations in human colorectal cancer. Cancer Res. 65, 4562-4567 (2005).

17. Kang, S., Bader, A. G. \& Vogt, P. K. Phosphatidylinositol 3-kinase mutations identified in human cancer are oncogenic. Proc. Natl Acad. Sci. USA 102, 802-807 (2005).

18. Samuels, Y. et al. Mutant PIK3CA promotes cell growth and invasion of human cancer cells. Cancer Cell 7, 561-573 (2005).

19. Zhang, Y. et al. A pan-cancer proteogenomic atlas of PI3K/AKT/mTOR pathway alterations. Cancer Cell 31, 820-832 e823 (2017).

20. Lindhurst, M. J. et al. Mosaic overgrowth with fibroadipose hyperplasia is caused by somatic activating mutations in PIK3CA. Nat. Genet. 44, 928-933 (2012).

21. Venot, Q. et al. Targeted therapy in patients with PIK3CA-related overgrowth syndrome. Nature 558, 540 (2018).

22. Park, D. et al. Endogenous bone marrow MSCs are dynamic, fate-restricted participants in bone maintenance and regeneration. Cell Stem Cell 10, 259-272 (2012).

23. $L i, H$. et al. miR-216a rescues dexamethasone suppression of osteogenesis, promotes osteoblast differentiation and enhances bone formation, by regulating c-Cbl-mediated PI3K/AKT pathway. Cell Death Differ. 22, 1935-1945 (2015).

24. Liu, X. et al. Lifelong accumulation of bone in mice lacking Pten in osteoblasts. Proc. Natl Acad. Sci. USA 104, 2259-2264 (2007).

25. Ye, C. et al. Extracellular IL-37 promotes osteogenic differentiation of human bone marrow mesenchymal stem cells via activation of the PI3K/AKT signaling pathway. Cell Death Dis. 10, 753 (2019).

26. Caplan, A. I. Adult mesenchymal stem cells for tissue engineering versus regenerative medicine. J. Cell Physiol. 213, 341-347 (2007).

27. Dai, Q. et al. mTOR/Raptor signaling is critical for skeletogenesis in mice through the regulation of Runx2 expression. Cell Death Differ. 24, 1886-1899 (2017).

28. Yu, J. S. \& Cui, W. Proliferation, survival and metabolism: the role of PISK/AKT/ mTOR signalling in pluripotency and cell fate determination. Development 143, 3050-3060 (2016). 